\title{
Application and Outlook of In-situ Combustion for Developing Heavy Oil Reservoir
}

\author{
ZHANG Xishun ${ }^{1,}$, , LIN Chunqing ${ }^{2}$, GU Liming ${ }^{3}$, XIA Yinghu $^{4}$, GUO Zhaoxia ${ }^{5}$, \\ SHI Junfeng ${ }^{1}$, ZHAO Ruidong ${ }^{1}$, ZHANG Xin ${ }^{1}$, and CHEN Shiwen ${ }^{1}$ \\ ${ }^{1}$ Research Institute of Petroleum Exploration \& Development, CNPC, China \\ ${ }^{2}$ Research Institute of Petroleum Exploration \& Development, CNPC, China \\ ${ }^{3}$ Oil Development Department of Jidong Oilfield, CNPC, China \\ ${ }^{4}$ Supervision Station of Downhole Operation Department of Shanshan Oilfield, CNPC, China \\ ${ }^{5}$ Bidding Center of Huabei Oilfield, CNPC, China \\ ashunzi-2003@163.com
}

\begin{abstract}
Keywords: In-situ combustion, Heavy oil reservoir, Burning front, Ignition technique
Abstract. As the most potential development method of heavy oil reservoir, in-situ combustion has the maximal recovery factor due to its multiple displacement mechanisms. The paper presents the enhancing oil recovery mechanism of in-situ combustion through analyzing the combustion principle of crude oil in reservoir and displacement mechanism of burning front. Then the five key technologies of in-situ combustion were discussed primarily here on the basis of oil burning characteristic in reservoir, including the selecting criterion of in-situ combustion for heavy oil reservoir, ignition technique, monitoring technique of burning, combustion control technique of burning front, physical and mathematical simulation of in-situ combustion. From the discussion, present technique status was presented, the key problems were exposed, and the technique demands and long term potential were brought forward. As In-situ combustion being in the ascendant, the paper summarized its enhancing oil recovery mechanism, the techniques demanding and the outlook in the future, which provided the most advanced technologies and point out the developing direction about it.
\end{abstract}

\section{Introduction}

Heavy oil in reservoir buried in several kilometers depth has high viscosity which makes it flow difficult and results in a low recovery factor. So enhancing oil recovery(EOR) of heavy oil reservoir was one of the most important topics researchers concerned Injecting heat medium to heat oil and reduce viscosity was the popular method to develop heavy oil reservoir, including hot water flooding and steam injection. The former results in water fingering and water flush inevitably due to the great mobility ratio between oil and water and the later results in steam overlapping because of the low density of steam, which both result in a low recovery factor in developing heavy oil reservoir. Still, heat medium injection needs huge energy consumption ${ }^{[1]}$.

As a new EOR method, in-situ combustion was proposed and made great progress from the 30th last century. A fire lighter is needed and putted into the down hole of wellbore; air is injected continually for some day until the heated air firing the oil layer around wellbore ${ }^{[1-4]}$. Then air is injected according to the injection project, which makes the burst front driven ahead from injection well to production well, and then heated oil, distilled oil and cracked oil will flow into production well naturally. From theoretical analysis, the burst front could generate multiple displacement effects. No water fingering and steam overlapping would be brought since no heat medium injected, which results in higher recovery factor and shorten heat waste. Crude oil would be burned in in-situ combustion unavoidably, but the losses are mainly the components of bitumen and coke. In recent years, with some key techniques being broken through and heavy oil development being in the ascendant resulted from energy shortage, in-situ combustion applied to develop heavy oil reservoir rapidly in many countries. 


\section{Mechanism of in-situ combustion}

As the heavy components of crude oil, Bitumen and coke was left and being the fuel through distilled and thermal cracked by burning front. The oxygen in air injected continually and regularly maintains the burning front moving from injection well to production well. As the main component, distilled and cracked light oil was driven ahead. Steam and $\mathrm{CO}_{2}$ were given birth to through the burning between oxygen with the fuel, which can generate the two conventional EOR effects of steam driving and $\mathrm{CO}_{2}$ miscible flooding respectively. From injection well to production well, the reservoir can be divided into five zones which as burning zone, coke zone, steam zone, light oil zone and originality zone sequentially as the fig1. So, in-situ combustion have multiple effects of displacements such as steam driving, hydrocarbon miscible flooding and $\mathrm{CO}_{2}$ miscible flooding, which result in a higher recovery factor than other EOR method ${ }^{[3-6]}$.

Temperature distribution
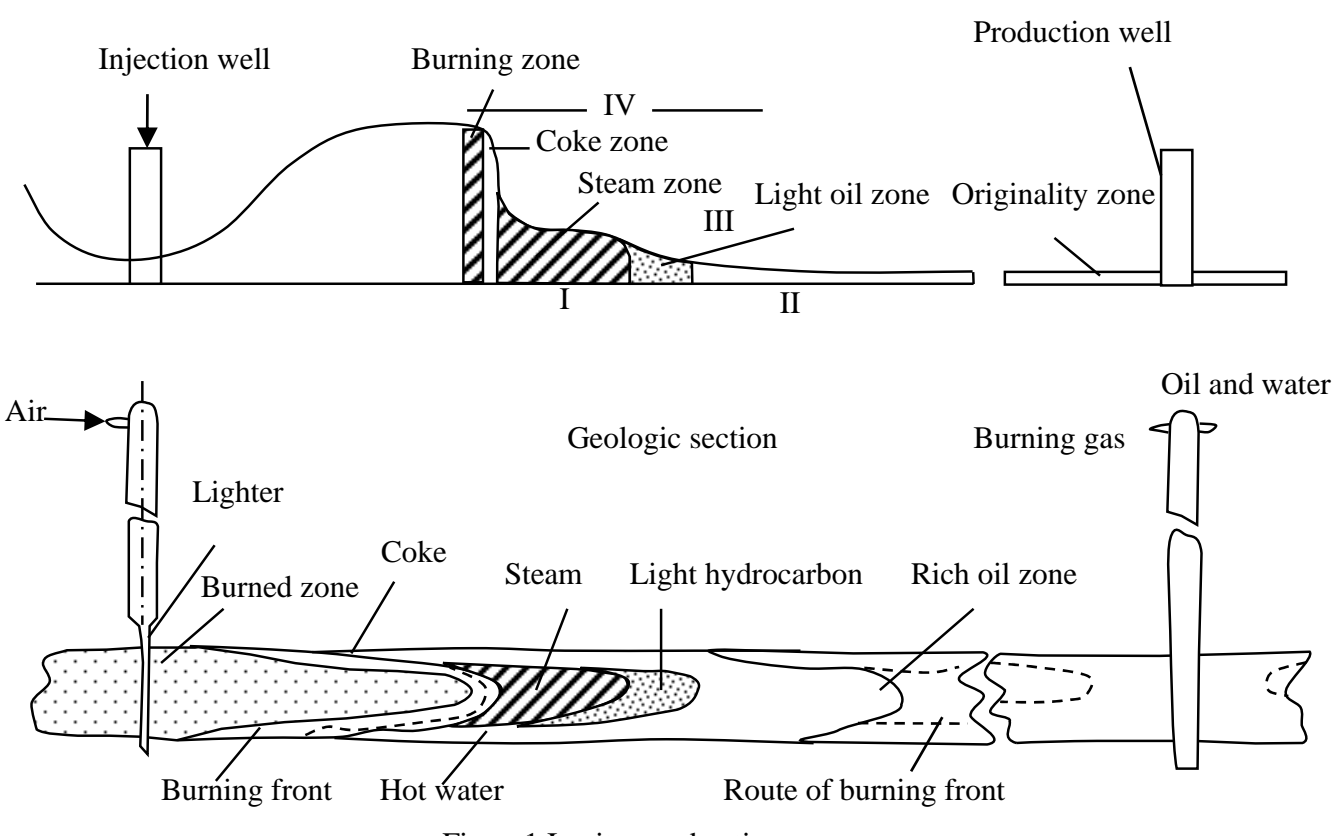

Figure1 In-situ combustion process

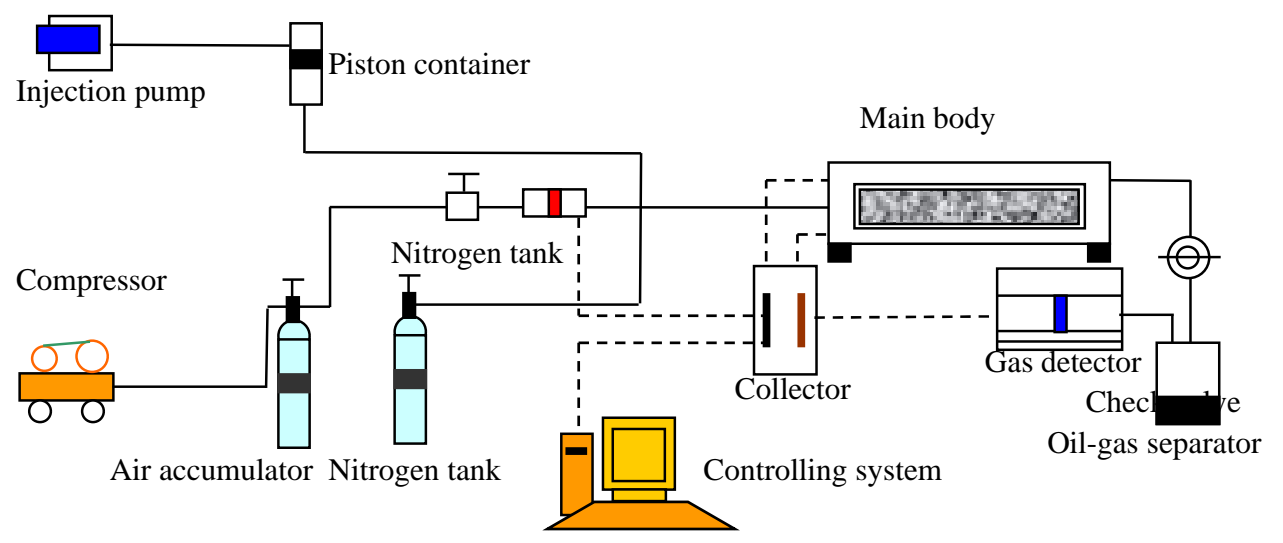

Figure2. The flow chart of 3D physical simulation

For the evolution behavior of crude oil in burning, thermo-gravimetry can be used to study the function mechanism. Different group and component in crude oil has different burning behavior, in which saturated hydrocarbon and aromatic hydrocarbon are in the extreme.

In general, the process of burning can be fallen into two phases: distillation phase and oxidation phase. The former generated in the temperature ranged $25 \sim 300{ }^{\circ} \mathrm{C}$, the later can be divided into three 
phases which as low temperature oxidation(LTO), middle temperature oxidation(MTO) and high temperature oxidation $(\mathrm{HTO})^{[6,7]}$.

As the lightest component, saturated hydrocarbon would burn firstly, which occurs in the phase of LTO and the loss be about in $89 \%$. The high components such as bitumen and coke lose very little in the phase. In the phase of MTO, the effect of saturated hydrocarbon decreased and the asphaltene and aromatic hydrocarbon began to bring into play. The chemical reactions are all gas phase reactions including the oxidation of cracked product, which produces the fuel of HTO. The product of MTO oxidized further and the HTO generated, in which bitumen and coke play the important roles and the loss accounts for $90 \%$. The reaction in HTO phase, a large amount heat was produced to burn coke, crack crude oil and heat saturated hydrocarbon, which were the EOR mechanism of in-situ combustion. In a word, saturated hydrocarbon has earlier and higher reactivity, which can be as the igniter and control the combustion front, bitumen and coke being the fuel, maintain the constancy of burning.

\section{Key techniques and its status}

From theoretical analysis to practical application, in-situ combustion must work out some key techniques including selecting criterion of candidate reservoir, firing technique, burning front monitoring and controlling, physical simulation and mathematical simulation.

Selecting criterion of candidate reservoir. Despite the face that in-situ combustion is one of the most scientific EOR methods, not all heavy oil reservoirs were suited to develop in it. As in-situ combustion being a high investment project, selecting criterion must be formulated before carry into execution, which bases on crude oil property, nature of rock and reservoir physical property. America Petroleum Institute (API), Gephen and Chuke etc. had presented some selecting criterions ${ }^{[4,5]}$ based on oil viscosity, reservoir thickness, reservoir depth, content of coke and so on. The values of these criterions are different for different researcher because they come from some successful example reservoirs of in-situ combustion. So, the values can not be applied generally.

D.V.Yannimaras introduced a selecting method ${ }^{[7]}$ through testing thermodynamics parameters of crude oil by accelerating rate calorimeter (ARC). ARC is the apparatus applied to study on reaction kinetics through adiabatic reaction, which can obtain the thermodynamics parameters of crude oil in a wide pressure range of 10.0 70.0MPa.

Arrhenius activation energy, index factor, reaction order, initial temperature and heat release degree can be tested by ARC on which we based to select the appropriate candidate reservoir to develop in in-situ combustion. Furthermore, if combustion tube testing combined to ARC, the selecting result would be more credible.

Ignition technique. Igniting the around reservoir and achieving stable burning is the first but the utmost step of in-situ combustion, which decides the effect of in-situ combustion. Ignition methods included self-ignited in reservoir and ignited manually. The former had few or no application because it's just appropriate to specially reservoir and the time of ignition is very long. For the later, electric heater ignition is the most general and advanced technique, in which clubbed form heater is popular. $\mathrm{Li}$ Youping, Li Shulan etc. invented SL-I high power electric heater ignition ${ }^{[8,9]}$. When ignition device was selected, corresponding ignition parameters must be decided reliably. Tetama and Haitama developed the calculated formula of igniting time through the energy balance of LTO. Li Shulan presented the predication model of air injecting rate, electrical parameters, and temperatures through simulating temperature and pressure distribution.

Monitoring and controlling techniques of burning front. After reservoir was ignited, monitoring and controlling burning was the significant task which maintains the burning front burning uniformly and driving firmly and results in a high recovery factor.

As the burning front driving ahead radically, different burning phase needs different air injection rate, which makes the burning stably and the sweep efficiency spread. Nelson and Mcniel developed a air injection model ${ }^{[9]}$, which has been accepted by researchers generally. The model divided the injection course into three stages: primary stage, steady stage and final stage. For the primary stage, the air injection rate can be calculated by linear function. When the injection rate increases to the 
maximum value, then injection keeps steadily in the value that is the steady stage. The steady stage maintains sited time, and then the injection rate can be calculated by linear descend function. The key parameters needed determined are the maximum value of injection rate and the time of steady stage kept.

Monitoring the burning front dynamically is the basis of analyzing and adjusting injection project. Dynamic monitoring of in-situ combustion can be classed into two parts. One part is production status monitoring of injection and production wells including pressures and temperatures of the both wells, injection rate, oil production rate, gas production rate etc. Furthermore, production cross-sectional testing should be considered through steady and unsteady state well testing. The monitoring parameters are same to water flooding wells', but should be obtained under high temperature condition. The other part is monitoring property and components of production liquid, especially the production gas components. Through the monitoring data, the burning condition can be analyzed and injection project can be adjusted in time.

Physical simulation technique of in-situ combustion. As the investment of in-situ combustion being huge, every in-situ combustion project needs laboratory physical simulation before execution. The physical simulation in laboratory includes combustion kettle testing, one dimension combustion tube testing and 3D combustion testing ${ }^{[10,11]}$.

Mathematical simulation of in-situ combustion. Mathematical model is needed to simulate the burning and production course of in-situ combustion since the mechanism is very complex and can be discovered by 3D combustion testing. Primitively, a three phases and five components (oxygen, nitrogen, $\mathrm{CO}_{2}$, water and oil) model had been developed and to simulate the flow of liquid and thermal transmission. With the advancing of mathematical theory and computer technology, CMG company developed a combustion simulator which consider many of components to simulate the flow law of liquid, thermal transmission, burning, gasification, cracking and cooling ${ }^{[12]}$. Furthermore, the displacement and production course can be simulated and predicted. For mathematical simulation, the testing data from physical simulation is very significant to the accuracy of predicted result.

\section{Technologies demand and long term potential}

From the analysis, all the key technologies above need improve if in-situ combustion wants to be a widespread development method for heavy oil reservoir ${ }^{[7-12]}$.

I For selecting criterion of candidate reservoir, at present the considered factors were few and empirical. More factors should be considered and comprehensive assessment method such as fuzzy mathematics should be applied to develop a scientific selecting criterion.

I For firing technique, ignition device appropriated for deep well, high temperature well and high water ratio well should be developed.

I As the high temperature and poly component in-situ combustion being, monitoring technique should break through the limitation of high temperature.

I With the development of 3D physical simulation, more influencing factors should be considered to make the simulation more analogous to practical combustion, such as physical similarity, burning front monitoring etc.

\section{Conclusions}

The paper presented the enhancing oil recovery mechanism of in-situ combustion through analyzing the combustion principle of oil in reservoir and displacement mechanism of burning front. Five zones were classed as burning zone, coke zone, steam zone, light oil zone and originality zone sequentially.

Five key technologies of in-situ combustion were discussed significantly here on the basis of heavy oil combustion principle in reservoir, including the selecting method of in-situ combustion reservoir, firing technique, monitor technique of burning front, combustion control technique, physical and mathematical simulation of in-situ combustion. 
Technologies demand and long term potential were pointed out based on the analysis of key techniques.

\section{References}

[1] Guan Wenlong, Wu Shuhong, Liang Jinzhong, et al. The research on engineering risk in combustion assisted gravity drainage based on indoor experiment. Journal of Southwest Petroleum University. 2009, 31(4), 67-72.

[2] Adegbesan K O, et a1. Low Temperature Oxidation Kinetic Parameters for In-Situ Combustion: Numerical Simulation. SPE 12004.

[3] Binder G G, et a1. Scaled Model Tests of In Situ Combustion in Massive Unconsolidated Sands : World Pet. Cong. Maxico City, 1967: 477-485

[4] Zhao Dongwei, Jiang Haiyan, Zhang qi. Dry combustion physical modeling of in-situ combustion. Oi1Drilling \& Production Technology, 2005, 27(1): 36-39.

[4] GRABOWSKI Janusz W, VINSOME Paul K, LIN Ran C, et al. A fully implicit general finite-difference thermal model for in-situ combustion and stearn. SPE8396, 1979.

[5] LIN C Y, CHEN W H, CULHAM W E. New kinetic thermal cracking of crude oil in in-situ combustion processes. SPE 13074, 1987.

[6] ONYEKENWOMO, PANDE K, RAMEY H J, et a1. Experimental and simulation studies of laboratory in-situ combustion recovery. SPE 15090, 1996.

[7] DU Dianfa, YAO Jun. Effect of development parameters on in-situ cornbustion after iniecting steam in Le an oil Field. Journd of the University of Petroleum, China, 2003, 27(2): 47-50.

[8] Kumar M. Simulation of laboratory in-situ combustion data and effect of process variation. SPE 16027, 1987.

[9] Crookstion R B, Culham W E. The numerical simulation model for thermal recovery process. SPEJ, 1979.

[10]Penberthy Jr W L, Ramey. Design and operation of laboratory combustion tubes. SPE 1290, 1966.

[11]Coats R, Lorimer S, Ivory J. Experimental and numerical simulations of a novel top down in-situ combustion process. SPE 30295, 1995.

[12] Jia Zhenyuan et al. Formation and distribution of hydrocarbon in carbonate. Beijing: Petroleum Industry Press, 1 989: 45-63. 